\title{
Unique water-water coordination tailored by a metal surface
}

Schiros, T.; Andersson, Klas Jerker; MacNaughton, J.; Gladh, J.; Matsuda, A.; Öström, H.; Takahashi, O.; Pettersson, L. G. M.; Nilsson, A.; Ogasawara, H.

Published in:

Journal of Chemical Physics

Link to article, DOI:

$10.1063 / 1.4809680$

Publication date:

2013

Document Version

Publisher's PDF, also known as Version of record

Link back to DTU Orbit

Citation (APA):

Schiros, T., Andersson, K. J., MacNaughton, J., Gladh, J., Matsuda, A., Öström, H., Takahashi, O., Pettersson, L. G. M., Nilsson, A., \& Ogasawara, H. (2013). Unique water-water coordination tailored by a metal surface. Journal of Chemical Physics, 138(23), 234708. https://doi.org/10.1063/1.4809680

\section{General rights}

Copyright and moral rights for the publications made accessible in the public portal are retained by the authors and/or other copyright owners and it is a condition of accessing publications that users recognise and abide by the legal requirements associated with these rights.

- Users may download and print one copy of any publication from the public portal for the purpose of private study or research.

- You may not further distribute the material or use it for any profit-making activity or commercial gain

- You may freely distribute the URL identifying the publication in the public portal 


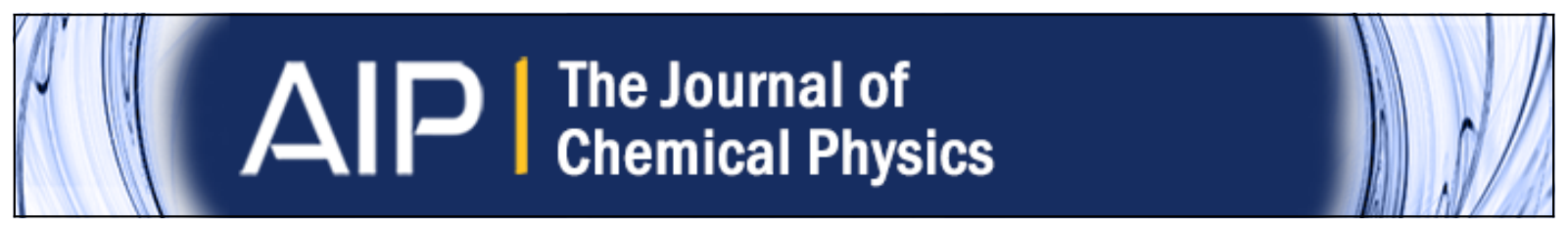

\section{Unique water-water coordination tailored by a metal surface}

T. Schiros, K. J. Andersson, J. MacNaughton, J. Gladh, A. Matsuda, H. Öström, O. Takahashi, L. G. M.

Pettersson, A. Nilsson, and H. Ogasawara

Citation: The Journal of Chemical Physics 138, 234708 (2013); doi: 10.1063/1.4809680

View online: http://dx.doi.org/10.1063/1.4809680

View Table of Contents: http://scitation.aip.org/content/aip/journal/jcp/138/23?ver=pdfcov

Published by the AIP Publishing

\section{AlP Re-register for Table of Content Alerts}




\title{
Unique water-water coordination tailored by a metal surface
}

\author{
T. Schiros, ${ }^{1,2, a)}$ K. J. Andersson, ${ }^{3}$ J. MacNaughton, ${ }^{1}$ J. Gladh, ${ }^{2}$ A. Matsuda, ${ }^{2,4}$ H. Öström, ${ }^{2}$ \\ O. Takahashi, ${ }^{5}$ L. G. M. Pettersson, ${ }^{2}$ A. Nilsson, ${ }^{1,2}$ and H. Ogasawara ${ }^{1, b)}$ \\ ${ }^{1}$ Stanford Synchrotron Radiation Lightsource, SLAC National Accelerator Laboratory, 2575 Sand Hill Road, \\ Menlo Park, California 94025, USA \\ ${ }^{2}$ Department of Physics, Stockholm University, AlbaNova University Center, S-10691 Stockholm, Sweden \\ ${ }^{3}$ Center for Individual Nanoparticle Functionality (CINF), Department of Physics, Technical \\ University of Denmark, Fysikvej 312, DK-2800 Kgs. Lyngby, Denmark \\ ${ }^{4}$ Research Center for Materials Science, Nagoya University, Furo, Chikusa, Nagoya, Aichi 464-8602, Japan \\ ${ }^{5}$ Department of Chemistry, Hiroshima University, Higashi-Hiroshima 739-8526, Japan
}

(Received 1 February 2013; accepted 23 May 2013; published online 21 June 2013)

\begin{abstract}
At low coverage of water on $\mathrm{Cu}(110)$, substrate-mediated electrostatics lead to zigzagging chains along [001] as observed with STM [T. Yamada, S. Tamamori, H. Okuyama, and T. Aruga, "Anisotropic water chain growth on $\mathrm{Cu}(110)$ observed with scanning tunneling microscopy" Phys. Rev. Lett. 96, 036105 (2006)]. Using x-ray absorption spectroscopy we find an anomalous lowenergy resonance at $\sim 533.1 \mathrm{eV}$ which, based on density functional theory spectrum simulations, we assign to an unexpected configuration of water units whose uncoordinated $\mathrm{O}-\mathrm{H}$ bonds directly face those of their neighbors; this interaction repeats over trough sites with enhanced electron density and is analogous to the case of a hydrated electron. () 2013 AIP Publishing LLC. [http://dx.doi.org/10.1063/1.4809680]
\end{abstract}

\section{INTRODUCTION}

The structure of water at metal surfaces results from the balance between the water-water and water-metal bond strength which is determined by a number of effects, including electrostatic enhancement and creation of electric fields at the surface through proton orientation and ordering, the degree of lattice match to the metal surface and influence of electronic and geometric structure of the given surface. These factors influence the structure and bonding of water at metals and determine barriers to dissociation, interaction with multilayer ice, and wetting properties with enormous implications for a number of environmentally and technologically important reactions, from biology to materials science to electrocatalysis and corrosion. $^{1-4}$

Numerous experimental studies, particularly with STM, have revealed complicated variations in the observed or proposed structure of the low-coverage phase of water at different metal surfaces, such as lacey rosettes ${ }^{5}$ and honeycomb island structures ${ }^{6}$ on $\operatorname{Pd}(111)$, from dimers ${ }^{7}$ to non-hydrogen-bonded water molecules on $\mathrm{Ni}(110),{ }^{8}$ hydrogen-bonded cyclic water hexamers at low coverage and low temperatures on $\mathrm{Cu}(111), \mathrm{Ag}(111)$, and $\mathrm{Ru}(0001),{ }^{9-11}$ zigzag chain or cyclic forms of clusters at low coverage and low temperatures on $\mathrm{Cu}(110)^{12,13}$ and a proposed chaingrowth of hexamer ${ }^{12}$ or pentamer ${ }^{14}$ units on $\mathrm{Cu}(110)$.

The interaction of water with the $\mathrm{Cu}(110)$ surface is a particularly interesting case because, although water adsorbs molecularly on this surface at low temperatures, ${ }^{15}$ the energy

\footnotetext{
a) Present address: Energy Frontier Research Center, Columbia University, New York, New York 10027, USA.

b) Author to whom correspondence should be addressed. Electronic mail: hirohito@slac.stanford.edu
}

difference between intact and dissociative adsorption is very small. ${ }^{16}$ This may be partly responsible for the preeminence of $\mathrm{Cu}$ as the primary catalyst for the water-gas shift reaction, which plays an important role in the production of high purity hydrogen for fuel cells ${ }^{17}$ as well as industrially important chemicals such as ammonia and methanol. ${ }^{18,19}$

Here we combine x-ray absorption spectroscopy (XAS), $\mathrm{X}$-ray photoelectron spectroscopy (XPS), and sum-frequency generation (SFG) with density functional theory (DFT) calculations to investigate the low-coverage phase of water adsorbed on $\mathrm{Cu}(110)$. We find that at $\sim 0.3 \mathrm{ML}$, water molecules are arranged into clusters, in which each water molecule acts as a single $\mathrm{H}$-bond acceptor and a single $\mathrm{H}$-bond donor. We also identified the origin of linkage between clusters forming the observed chain form. ${ }^{12}$ The points of contact between neighboring units are stabilized with an unexpected water structure, where the uncoordinated $\mathrm{O}-\mathrm{H}$ bonds protruding from neighboring units point toward each other atop the troughs in the open (110) surface. This type of proton orientation is similar to the Bjerrum defect, ${ }^{20}$ which exists at extremely low concentration in ice due to high energetic cost. Such a defect has been proposed for hydroxyl groups in the mixed water/hydroxyl layer on $\mathrm{Cu}(110) .{ }^{21}$ In the present study on intact adsorbed water, the spectral fingerprint of this unique opposing proton structure appears as an intense lowenergy resonance in the XAS spectrum. As the coverage increases, attractive interaction between water units switches from the substrate-mediated Bjerrum defect to hydrogenbonding (H-bonding) and the low-energy XAS resonance disappears. The stability of the unique low-coverage structure for water on $\mathrm{Cu}(110)$ is rationalized using both molecular orbital and electrostatic considerations and a connection to hydrated electrons is made. 


\section{METHODS}

The XPS and XAS experiments were performed at undulator beamline I511 at the MAX II synchrotron radiation facility in Lund, and at beamline 5-1 at the Stanford Synchrotron Radiation Lightsource (SSRL). Both surface science end stations were equipped with Gammadata-Scienta electron analyzers. A typical operating pressure lower than $1 \times 10^{-10}$ Torr was achieved during experiment. An incidence angle of $5^{\circ}$ for the incoming radiation was used. XPS and XAS spectra were obtained using total energy resolution better than 0.3 and $0.1 \mathrm{eV}$, respectively. Two crystals were mounted with the [001] direction perpendicular to each other in order to enable recording angle-dependent spectra with the E-vector along the [1-10], [001], and [110] directions. The XAS was recorded in Auger electron yield (AEY) mode at I511 by collecting the Auger electrons resulting from oxygen KVV transitions with a Gammadata-Scienta SES-200 electron energy analyzer. The XAS was recorded in partial electron yield (PEY) mode at 5-1 using a micro-channel-plate detector with retarding grids. ${ }^{22}$ The retarding voltage was set to $-320 \mathrm{~V}$. Beamdamage was avoided by minimizing the photon dose and scanning the sample to always measure a fresh spot and by utilizing $\mathrm{D}_{2} \mathrm{O}$ to avoid beam-induced dissociation of water. ${ }^{15}$ The absence of the O1s XPS feature assignable to hydroxyl $(\sim 531 \mathrm{eV})$ confirmed no beam damage in these measurements.

SFG experiments were carried out using a broadband SFG setup with a $1 \mathrm{kHz}$ Ti:sapphire laser providing $800 \mathrm{~nm}$ laser pulses with a pulse energy of $2.1 \mathrm{~mJ}$ and a duration of $50 \mathrm{fs}$. The laser pulses were converted in an optical parametric amplifier to infrared pulses with a spectral width of $\sim 250 \mathrm{~cm}^{-1}$ (FWHM) and pulse energy up to $10 \mu \mathrm{J}$. The remaining $800 \mathrm{~nm}$ light was spectrally narrowed down to $\sim 5 \mathrm{~cm}^{-1}$ and both pulses were focused to $\sim 500 \mu \mathrm{m}$ and overlapped at grazing incidence on the sample to generate the sum-frequency signal, which was recorded using a spectrograph equipped with an intensified CCD detector. The spectra were frequency calibrated using the vibrational resonance of $(2 \times 1)$ adsorbed $\mathrm{CO}$ giving an uncertainty of $\pm 5 \mathrm{~cm}^{-1}$ in absolute vibrational frequency.

$\mathrm{The} \mathrm{Cu}(110)$ crystals were cleaned by cycles of Argon ion sputtering and annealing to $900 \mathrm{~K}$ until a well-ordered LEED pattern was obtained and XPS showed no contamination $(\mathrm{C}, \mathrm{O}<0.1 \% \mathrm{ML})$. The monolayer of water was obtained by dosing $\mathrm{D}_{2} \mathrm{O}$ (utilized in order to minimize beam damage in the synchrotron-based measurements) at $100 \mathrm{~K}$ and then annealing multilayer water to $140 \mathrm{~K}$. Water was stored in a glass container connected to the gas delivery system and was initially cleaned by multiple freeze-pump-thaw cycles, then boiled under vacuum at room temperature to remove dissolved oxygen and carbon dioxide; purity was checked by mass spectrometry prior to experiments. The water coverage was estimated from XPS. There is an XPS binding-energy shift between the multilayer and the monolayer water. ${ }^{23}$ The monolayer of water was obtained by annealing a water multilayer to $140 \mathrm{~K}$, and the absence of the multilayer XPS peak was confirmed. In the SFG experiment both $\mathrm{D}_{2} \mathrm{O}$ and $\mathrm{H}_{2} \mathrm{O}$ were used and the uptake was recorded after dosing small

amounts of water at $105 \mathrm{~K}$ and the coverages were calibrated using thermal desorption.

All calculations were performed within the framework of density functional theory (DFT). The StoBe-deMon code ${ }^{24}$ was used to optimize cluster models, compute XAS spectra based on the half-core-hole transition potential approach ${ }^{25,26}$ as well as XPS binding energies and total energy. A description of the basis sets and how the electronic structure was represented has been provided in Ref. 27. The computed XAS oscillator strengths were broadened with Gaussians of increasing full width at half-maximum (fwhm) and the absolute energy scale was determined through Delta Kohn-Sham calculations; ${ }^{28}$ the computed shift gave a good match with the experimental resonance for the in-plane H-bond network at $540 \mathrm{eV}$.

\section{RESULTS AND DISCUSSION}

Our key experimental result is given in the central panels of Fig. 1 where we compare the in- and out-of-plane excited XAS of low-coverage $(0.3 \mathrm{ML})$ water on $\mathrm{Cu}(110)$ with water at a similar coverage on $\mathrm{Ru}(0001)$ where, in the latter case, $2 \mathrm{D}$ clusters are known to form; by tuning the electric field vector of the incoming x-ray beam perpendicular or parallel to the plane of the surface, the directionality of chemical bonds can be distinguished. As a reference we show the out-of-plane XAS spectrum of the surface of ice ${ }^{29}$ where the strong resonance at $535 \mathrm{eV}$ (A2 in the figure) has been assigned as due to non-H-bonded $\mathrm{O}-\mathrm{H}$ bonds at the interface. The same feature (A2) is found also for the $2 \mathrm{D}$ clusters of water on $\mathrm{Ru}(0001)$ in the in-plane spectrum assigned to non-H-bonded $\mathrm{O}-\mathrm{H}$ bonds at the edges of the flat clusters. ${ }^{11}$ For low-coverage water on $\mathrm{Cu}(110)$, on the other hand, we find a strong resonance (A1) in the in-plane spectrum at significantly lower energy, $533.1 \mathrm{eV}$. We note that the sharp XAS pre-edge resonance in the

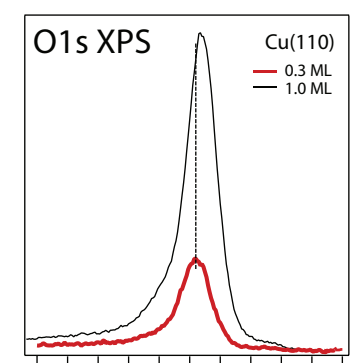

538536534532530528 Binding Energy/eV

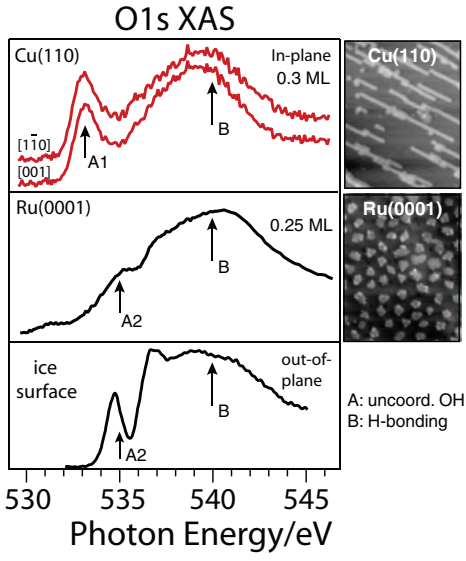

FIG. 1. O1s XPS (left) and in-plane XAS for (center, top) 0.3 ML $\mathrm{D}_{2} \mathrm{O} / \mathrm{Cu}(110)$, (center, middle) $0.25 \mathrm{ML} \mathrm{D}_{2} \mathrm{O} / \mathrm{Ru}(0001)^{47}$ and (center, bottom) out-of-plane XAS for ice surface. ${ }^{29}$ Characteristic features associated with $\mathrm{A}$ : uncoordinated $\mathrm{O}-\mathrm{H}$ bond at $535 \mathrm{eV}$ and $\mathrm{B}$ : hydrogen-bonding between water molecules at $540 \mathrm{eV}$ are denoted with arrows. A1 $(533.1 \mathrm{eV})$ is assigned to uncoordinated $\mathrm{O}-\mathrm{H}$ bonds in a unique, hitherto unobserved bonding arrangement. Far right: STM images showing that water assembles into chains at low coverage on $\mathrm{Cu}(110)^{12}$ and aggregates into isolated water clusters on $\mathrm{Ru}(0001){ }^{46}$ 
spectrum for the ice surface, (at $535 \mathrm{eV}$ ), which corresponds to uncoordinated $\mathrm{O}-\mathrm{H}$ bonds, is not observed in the out-ofplane spectrum for either $\mathrm{Ru}(0001)$ (at $535 \mathrm{eV}$ ) or $\mathrm{Cu}(110)$ (at $533.1 \mathrm{eV})$ at low coverage. This supports both for $\mathrm{Ru}(0001)$ and $\mathrm{Cu}(110)$ a water phase at low coverage with only O-bonding interaction with the metal and the molecular plane parallel to the surface as suggested by XPS (this work) and STM. ${ }^{12,13}$ O-bonded water molecules have also been shown to characterize the low-coverage phase of water on $\mathrm{Ni}(110)$, $\mathrm{Pd}(111), \mathrm{Cu}(111), \operatorname{Ag}(111)$, and $\mathrm{Ru}(0001){ }^{7-9,30,31}$

The individual water molecule has available two empty levels of valence character: the $\mathrm{O}-\mathrm{H}$ anti-bonding $4 \mathrm{a}_{1}$ and $2 \mathrm{~b}_{2}$ states, with $4 \mathrm{a}_{1}$ lowest in energy. When both $\mathrm{O}-\mathrm{H}$ bonds of a molecule are involved in $\mathrm{H}$-bonding the $p$-character in these states gets pushed up in energy to form the post-edge feature at $540 \mathrm{eV}$ (B in the central panels of Fig. 1) while if the $\mathrm{H}$-bonding is asymmetric with one bonded and one uncoordinated the $\mathrm{H}$-bonded contributes to the post-edge while the uncoordinated $\mathrm{O}-\mathrm{H}$ bond gives rise to the localized state ${ }^{32}$ seen as the pre-edge (A2 in Fig. 1); ${ }^{11,26,33-36}$ the presence of the $540 \mathrm{eV}$ resonance in all spectra indicates that water molecules immediately cluster through $\mathrm{H}$-bonding on $\mathrm{Cu}(110)$ at $100 \mathrm{~K}$. The pre-edge state at $535 \mathrm{eV}$ at the ice surface and on $\mathrm{Ru}(0001)$ is shifted up by $\sim 1 \mathrm{eV}$ compared to $4 \mathrm{a}_{1}$ in gas phase, but the corresponding state in low-coverage $\mathrm{H}_{2} \mathrm{O}$ on $\mathrm{Cu}(110)$ is shifted down by nearly $1 \mathrm{eV}$ compared to gas phase. The question is then what can cause the stabilization of this state relative to the gas phase?

STM measurements show patterned structures for water at $\mathrm{Cu}(110)$ already at low coverage, which both Yamada et al. ${ }^{12}$ and Carrasco et al. ${ }^{14}$ proposed to consist of water molecules arranged into chains that zigzag across the rows along the [001] direction (see insert in Fig. 1). However, while Yamada et al. described the water clusters in the chains as hexameric units of flat-lying water, ${ }^{12}$ Carrasco and co-workers concluded the 1D ice chains were built from face-sharing pentagons, with the water molecule occupying the trough site oriented with a hydrogen atom toward the surface. ${ }^{14}$ Although there is some debate over the structural details of the water cluster units, it is clear that these water clusters form chains on $\mathrm{Cu}(110)$ at low coverage.

The O1s XPS for 0.3 and 1.0 ML $\mathrm{D}_{2} \mathrm{O}$ on $\mathrm{Cu}(110)$ are shown in the left part of Fig. 1. The single XPS peak excludes the presence of hydroxyl groups from water dissociation, ${ }^{15}$ suggesting that this phase consists of molecularly adsorbed water, in agreement with the STM interpretation of Yamada et al. ${ }^{12}$ Using DFT we computed the $\mathrm{O} 1 \mathrm{~s}$ binding energy for different structures built from intact molecules such as dimers to trimers to hexamers, with water molecules either O-bonded to the surface or in H-up or H-down configurations; ${ }^{27,37}$ apart from structures with $\mathrm{H}$-up or H-down waters, which showed a too large XPS shift compared to O-bonded water, these, as well as isolated pentamers and hexamers with all molecules in O-bonded configuration, were all found consistent with the narrow XPS spectrum.

The flat-lying water molecules in the cyclic hexamer structure proposed by Yamada and co-workers ${ }^{12}$ and in the structure with face-sharing pentagons proposed by Carrasco and co-workers ${ }^{14}$ also matched the XPS spectrum. The max- imum XPS binding energy shifts for both the model incorporating the Bjerrum defect structure identified with XAS and the structure reported by Carrasco et al. are $0.2-0.4 \mathrm{eV}$ (see Table $1^{38}$ ). The structure results from a reorientation of the water molecules so that the uncoordinated $\mathrm{O}-\mathrm{H}$ bonds on the troughs of the (110) surface are directed slightly downward, while the oxygen tilts away. This geometry is favored by the electrostatics of the corrugated surface that result from the Smoluchowski effect; ${ }^{39}$ specifically, a loss of charge along the rows and build-up of charge in the troughs. The chargedepleted rows favor flat-lying water molecules at top sites, while the troughs prefer to interact with the positive end of the water dipole. ${ }^{23}$

Additional information can be obtained from vibrational spectroscopy, which requires an out-of-plane dynamic dipole moment, which might seem contradictory to the flat geometry of water molecules at the surface. However, even homonuclear molecules have previously been observed to become dipole-active in both physisorption and chemisorption systems at metal surfaces. ${ }^{40-43}$ In these cases the dynamic dipole moment arises from the interaction between the molecule and the surface leading to oscillatory charge transfer during a vibrational cycle. In the same way the vibrational motion in a flat-lying species can induce an out-of-plane dynamic dipole moment. Using the StoBe code we computed the dynamic dipole moment out of the surface plane induced by the $\mathrm{O}-\mathrm{D}$ stretch of a flat-lying $\mathrm{D}_{2} \mathrm{O}$ monomer at the $\mathrm{Cu}(110)$ surface. The computed IR intensity is a factor $\sim 200$ smaller than that computed for adsorbed $\mathrm{CO}$, which is in good qualitative agreement with the relative absorption cross-sections for these adsorbed molecules in experiments, e.g., Refs. 27 and 44. A visible "free O-D" stretch resonance is thus rather a signature of uncoordinated $\mathrm{O}-\mathrm{D}$ bonds than of $\mathrm{O}-\mathrm{D}$ bonds necessarily pointing away from the surface and observed SFG spectra are thus perfectly consistent with a structure consisting of water molecules with the molecular plane parallel with the surface.

The left panel of Fig. 2 shows coverage-dependent vibrational SFG spectra obtained for $\mathrm{D}_{2} \mathrm{O} / \mathrm{Cu}(110)$ dosed at $105 \mathrm{~K}$ together with curve fits. The middle panel shows the SFG intensities without the non-resonant background and interference effects. At low coverage we observe, for $\mathrm{D}_{2} \mathrm{O}$, a sharp resonance at $2689 \mathrm{~cm}^{-1}$ together with broader resonant structures in the region around $2500-2600 \mathrm{~cm}^{-1}$, in close analogy with previously published IRAS data for low-coverage $\mathrm{H}_{2} \mathrm{O} / \mathrm{Cu}(110) .{ }^{14}$ An additional sharp resonance around $2400 \mathrm{~cm}^{-1}$ was reported in the literature. ${ }^{44}$ This resonance is not resolved in our SFG, possibly due to it being too close to the absorption band of $\mathrm{CO}_{2}$ in air, which reduces our SFG sensitivity in this spectral region. We did, however, observe the corresponding frequency-shifted resonance for adsorbed $\mathrm{H}_{2} \mathrm{O}$ at $3192 \mathrm{~cm}^{-1}$, shown in the right panel of Fig. 2.

As the $\mathrm{D}_{2} \mathrm{O}$ coverage is increased, a second sharp resonance at $2720 \mathrm{~cm}^{-1}$ appears and the intensity in the broad band at lower frequency grows. The resonance at $2720 \mathrm{~cm}^{-1}$ can be correlated with uncoordinated $\mathrm{O}-\mathrm{D}$ groups pointing into the vacuum for a $2 \mathrm{D}_{2} \mathrm{O}$ structure as the structure changes to a mixed H-down/H-up phase $;{ }^{27}$ the blueshift of the frequency compared to the low-coverage frequency of 


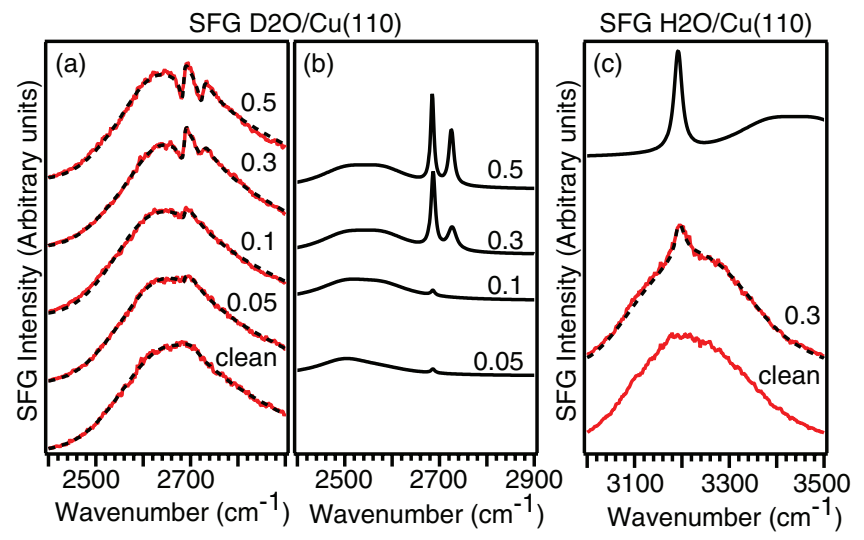

FIG. 2. (a) Coverage-dependent $\mathrm{D}_{2} \mathrm{O}$ SFG spectra (solid) of the O-D stretch region obtained after dosing at $105 \mathrm{~K}$ together with curve fits (dashed) from clean sample to $0.5 \mathrm{ML}$. The curve fits were obtained assuming that the intensity can be described by the square of the sum of a non-resonant susceptibility and 4 vibrational resonances, where the relative phases are taken into account. ${ }^{62}$ (b) Fitted SFG intensities after removal of phase and non-resonant background contributions. (c) The bottom panel shows the clean sample nonresonant background and SFG spectrum for $0.3 \mathrm{ML} \mathrm{H}_{2} \mathrm{O} / \mathrm{Cu}(110)$ (solid) together with curve fit (dashed). The top panel shows the fitted SFG intensity after removal of phase and non-resonant background contributions.

$2689 \mathrm{~cm}^{-1}$ shows directly that the non-H-bonded O-H/O-D bonds at low coverage experience a stabilizing interaction, albeit significantly weaker than through direct $\mathrm{H}$-bonding. The slight difference in coverage dependence compared to previously published IR data, which were recorded after flashing the $\mathrm{Cu}(110)$ crystal, is consistent with the temperaturedependent STM images recorded by Yamada et al. ${ }^{12}$ and indicate a stronger mix between $1 \mathrm{D}$ chains and 2D islands in our data. Can we reconcile the redshifted uncoordinated O-D resonance at $2689 \mathrm{~cm}^{-1}$ for the low coverage with the essentially flat-lying O-bonded $\mathrm{D}_{2} \mathrm{O}$ chains deduced from XAS?

XAS provides a sensitive probe of the H-bond network in water and over the last decade a library of XAS spectral fingerprints of water, as ice and liquid and adsorbed at metal surfaces has been established. ${ }^{11,27,29,33-37,45-47}$ In particular, uncoordinated $\mathrm{O}-\mathrm{H}$ bonds in asymmetrically $\mathrm{H}-$ bonded molecules have a spectral signature at $535 \mathrm{eV}$, while the $\mathrm{H}$-bonded $\mathrm{O}-\mathrm{H}$ bond produces a strong, broad resonance at $540 \mathrm{eV} \cdot{ }^{11,34-36,48-51}$ Intensity is observed at $535 \mathrm{eV}$ in the in-plane XAS spectrum for $\mathrm{Ru}(0001)$ due to uncoordinated $\mathrm{O}-\mathrm{H}$ bonds at the edges of the small isolated clusters that water aggregates into at low coverage on this surface (see Fig. 1, XAS and STM image). However, this feature is not discernible in the in-plane XAS on $\mathrm{Cu}(110)$ and instead another strong low-energy resonance is seen at $533.1 \mathrm{eV}$, which disappears at high coverage. ${ }^{27}$ This low-energy feature is seen for hydroxyl and atomic oxygen at metal surfaces, but the single XPS peak observed in Fig. 1 indicates that a purely molecular phase of water adsorbs at the $\mathrm{Cu}(110)$ surface. ${ }^{15}$ The resonance at $533.1 \mathrm{eV}$ is completely unique for a water hydrogenbonding system without the presence of hydroxyl. ${ }^{45,52}$ In fact, in our extensive structural search for a representation of water at low coverage on $\mathrm{Cu}(110)$, from dimers and trimers to pentamers and hexamers anchored over different adsorption sites,

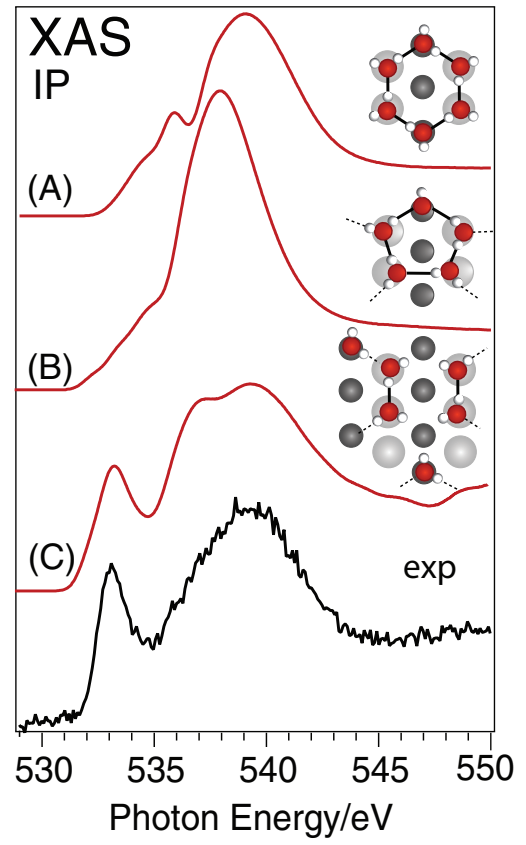

FIG. 3. Experimental and computed in-plane $\mathrm{XAS}^{38}$ for (A) hexamer, (B) pentamer chains proposed by Carrasco et al., ${ }^{14}$ and (C) a structure model of water chains characterized by Bjerrum defects stabilized by the Smoluchowski effect on the $\mathrm{Cu}(110)$ surface. The in-plane XAS corresponds to the sum of angle dependent XAS with the X-ray E-vector of the exciting light along the [1-10] and [001] directions. Computed spectra represent the summed contributions to the XAS signal from the different unique water molecules shown schematically in the insets; black lines represent the H-bond network, dashed lines indicate molecule in the schematic was H-bonded.

no simple traditional model could reproduce this experimental feature.

The computed in-plane XAS for a small cluster model including a single water hexamer on $\mathrm{Cu}(110)$ is shown in Fig. 3(A). The lowest energy feature in the computed spectrum appears at $535 \mathrm{eV}$ and is due to uncoordinated $\mathrm{O}-\mathrm{H}$ bonds parallel to the surface. ${ }^{11,27,33,35,36}$ While aspects of the experimental spectrum, particularly the broad resonance at $540 \mathrm{eV}$ due to $\mathrm{H}$-bonding in the hexamer, are well reproduced, the anomalous feature at $533.1 \mathrm{eV}$ is absent. Furthermore, no water chain arrangement of these subunits (dimer, pentamer, hexamer, etc.) with extended H-bonding between them could explain the origin of the $533.1 \mathrm{eV}$ resonance. In particular, the computed spectrum based on the structure proposed by Carrasco et al. ${ }^{14}$ (Fig. 3(B)) lacks intensity at $533.1 \mathrm{eV}$ and shows only a weak enhancement at $535 \mathrm{eV}$ from the water molecule at the end of the pentagon units. The post-edge position furthermore comes out at too low energy due to the long (1.85 $\AA$ ) average $\mathrm{H}$-bond distance; the post-edge results from excitations into H-bond-related anti-bonding states and is thus sensitive to H-bond distance through the principle of bonddistance-through-the-ruler in XAS. ${ }^{53,54}$

Based on these observations, we conclude that the $533.1 \mathrm{eV}$ XAS peak cannot be associated with the H-bonding network and anticipate that the feature may rather be due to a unique structure of the basic units, pentamers or hexamers, in the water chain at low coverage not fully covered by the pentamer model suggested by Carrasco et al. ${ }^{14}$ We use a flat-lying ring of water molecules as building block and 
consider how these units might be arranged in the zigzag pattern along the [001], with possible unexpected water-water interactions leading to the low-energy XAS feature. Since each water molecule in the cluster accepts a single $\mathrm{H}$-bond and donates a single $\mathrm{H}$-bond to the ring, uncoordinated hydrogen atoms (protons) will protrude from the edges of each hexamer or pentamer. To form extended chains, the protruding hydrogen atoms must face those of the neighboring units. In Fig. 3(C), we see that it is precisely this interaction that produces the low-energy resonance in the in-plane XAS. The resonance at $533.1 \mathrm{eV}$ appears in the XAS computed for these water molecules also in the absence of the $\mathrm{Cu}$ substrate, confirming that this feature is due exclusively to the unusual bonding arrangement of water molecules.

The bonding arrangements that produce the low-energy resonances are identified in Fig. 4, along with representative plots of the excited state orbitals involved in the XAS transition (the core-excited oxygen is shown in red). The large spatial extent of the excited state molecular orbital on the core-excited oxygen clearly encompasses the hydrogen atom protruding from the neighboring hexamer along the [001] (Fig. 4(a)). Since the opposing hydrogen atoms are positive, this interaction at the hexamer edges will lower the energy of the excited state. This is similar to the case of hydrated electrons, where uncoordinated $\mathrm{O}-\mathrm{H}$ bonds of water molecules near the cavity containing the electron rotate toward the center of the cavity to interact with and solvate the extra electron. ${ }^{55,56}$ In the present case, the negative troughs of the (110) surface play the role of the hydrated electron and electrostatically stabilize this unusual bonding configuration for water, which would otherwise be a high-energy defect as, e.g., in the case of Bjerrum defects in ice. ${ }^{20}$ This leads to an energy lowering of the XAS resonance associated with uncoordinated $\mathrm{O}-\mathrm{H}$ bonds from 535 to $533.1 \mathrm{eV}$ as well as to a redshift of the SFG vibrational frequency compared to uncoordinated $\mathrm{O}-\mathrm{H}$ bonds pointing away from the surface. Such low-energy states have also been observed in computed XAS of simulated liquid water structures ${ }^{57}$ obtained by partially neglecting charge repulsion when generating water structures from diffraction data ${ }^{58}$ in this case pockets with $\mathrm{O}-\mathrm{H}$ bonds pointing toward each other were also found to lead to computed very low-energy excitations. ${ }^{57}$

To investigate the role of the $\mathrm{Cu}(110)$ surface in the low coverage water structure we performed total energy calculations with and without the $\mathrm{Cu}(110)$ surface present. We obtain $+0.39 \mathrm{eV}$ hexamer-hexamer repulsion in the gas phase, but $-0.15 \mathrm{eV}$ hexamer-hexamer attraction when the Bjerrum defect is stabilized on the troughs of the $\mathrm{Cu}(110)$ surface where the electron density is high; note that a full relaxation of this structure using current DFT functionals leads to the protruding $\mathrm{O}-\mathrm{H}$ bonds rotating away and an energy lowering of the order $0.2 \mathrm{eV}$. The resulting structure does not show the lowenergy XAS peak at $533.1 \mathrm{eV}$ and is thus not supported by the XAS measurements. The energy-lowering is, however, small
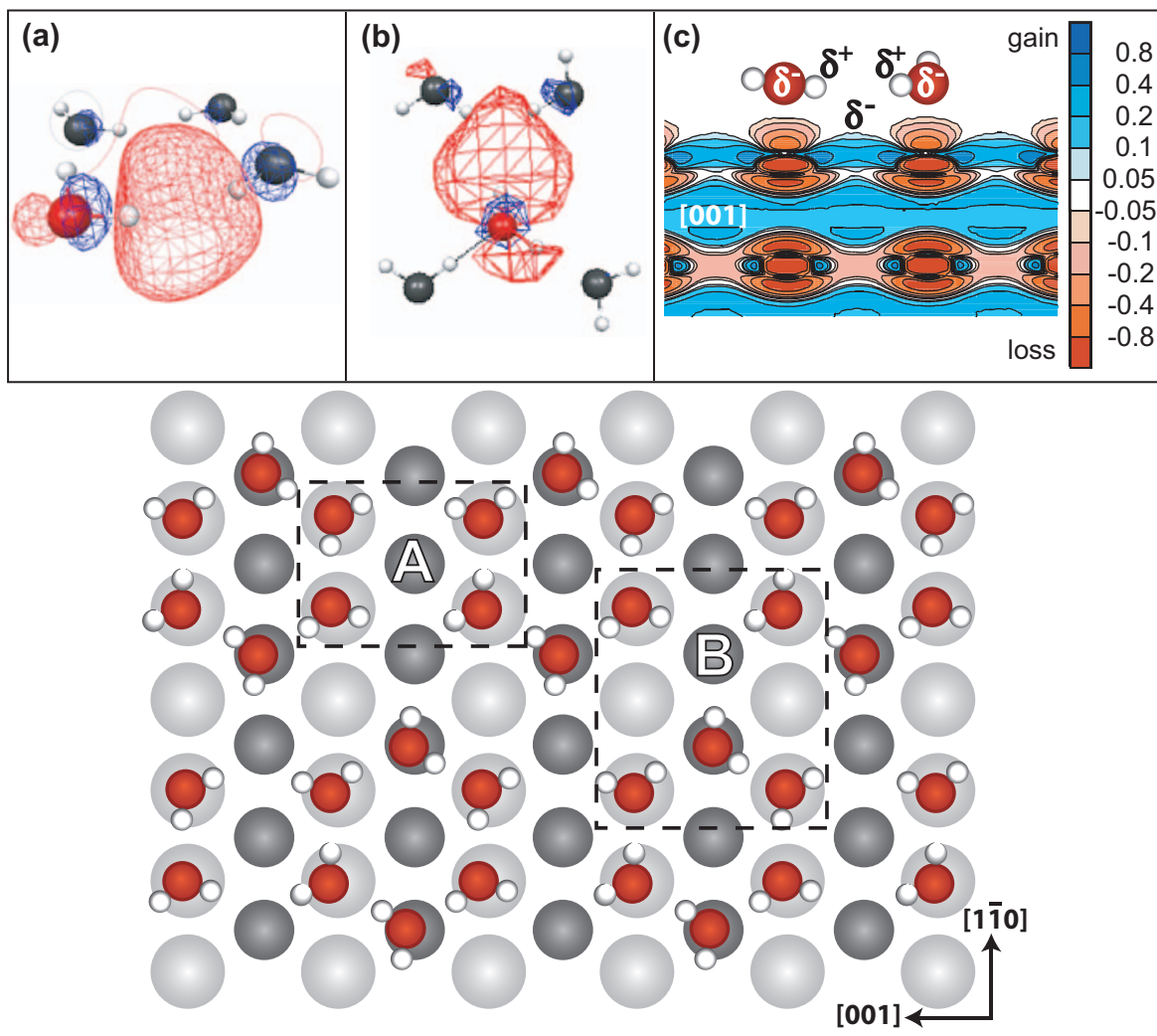

FIG. 4. Proposed structure for water on $\mathrm{Cu}(110)$ in the low coverage $(\theta<0.5 \mathrm{ML})$ regime, based on the bonding geometry that produces the low-energy XAS feature $\sim 533.1 \mathrm{eV}$ in the in-plane spectra (top). $\mathrm{Cu}$ atoms in the first rows are light grey and second-layer atoms are indicated with a darker color and smaller size. Interactions responsible for the XAS resonance, and orbital contour plots showing the spatial extent of the excited O orbital, which encompasses the protons of neighboring water molecules, are indicated in (a) and (b). In the contour plots, the core-excited $\mathrm{O}$ is indicated in red, while blue and red lines indicate different phases of the wave function. (c) Illustration of the Smoluchowski effect with charge depletion at the ridges and accumulation in the troughs, which provides an attractive electrostatic water-metal attraction and thus stabilizes the structure. 
enough that it is likely to be compensated by inclusion of, e.g., van der Waals interactions and quantum effects ${ }^{59,60}$ and we propose, based on the spectral evidence, that this Bjerrum defect-like configuration indeed is an important component in the chains at low coverage.

The polarization dependence of the experimental XAS shows that this interaction appears along both the [001] direction and the [1-10] direction. Fig. 4(b) shows that these conditions are satisfied by adjacent rows of identical clusters, situated across a zigzag pattern of uncoordinated $\mathrm{Cu}$ sites. Thus, the $\mathrm{Cu}(110)$ surface substrate stabilizes water cluster units in an unusual water environment with Bjerrum defects, in which protons face each other over the troughs. The reported STM images, parallel rows of protrusions oriented along the $[001]^{12,14}$ and four or six protrusions arranged in rectangular shapes, ${ }^{61}$ can thus be explained by water cluster units imaged as protrusions arranged by stabilized Bjerrum defects on the $\mathrm{Cu}(110)$ surface.

The stabilization is electrostatic in nature and related to the Smoluchowski effect, ${ }^{39}$ as described above. This is shown schematically in Fig. 4(c), which shows the charge density difference for the $\mathrm{Cu}(110)$ surface and the electrostatic attraction between the protons of opposing water molecules and the surface. Whereas the proton-proton interaction is repulsive, it is mitigated by the negative charge in the troughs, which spills over from top sites to smooth the potential energy at the corrugated surface. Between parallel rows of the water chain subunits (Fig. 4(b)), which are separated by a zigzag pattern of exposed $\mathrm{Cu}$ atoms alternating between rows and troughs, three protruding protons share the charge accumulated on a single $\mathrm{Cu}$ atom in the trough in a Bjerrum defect type structure. This defect is made possible by the oscillation of charge density at the surface, which occurs along the [001] but not along the [1-10], which explains the [001] chain orientation observed with STM. ${ }^{12,14}$ This can also explain the shape of water aggregates at very low coverage, in which four or six protrusions are arranged in rectangular shapes. ${ }^{61}$ The fact that only double rows of protrusions along the [1-10] are observed in the rectangular aggregates is explained by preferential development of Bjerrum defects along the [001]. Together, these factors provide the electrostatic driving force for water to take an unexpected turn in the low-coverage phase and generate an interesting structure hitherto not observed at a metal surface for a pure water adsorption phase.

\section{CONCLUSIONS}

In summary, water adsorbs on $\mathrm{Cu}(110)$ below $0.5 \mathrm{ML}$ to form chains of clusters oriented perpendicular to the rows. We here propose that the intersections that link the units into parallel chains are characterized by a novel water structure, similar to the Bjerrum defect in ice, where protons of opposing water molecules point toward one another. This structure can only exist in the sub-monolayer regime and, since it is largely stabilized by the Smoluchowski effect, will likely only appear for corrugated, open metal surfaces where the water-metal interaction is not so strong that clustering is unfavorable at low temperature. On flat surfaces like $\mathrm{Ru}(0001)$, in the absence of these surface-tailored electrostatics, water clusters remain isolated at low coverage.

\section{ACKNOWLEDGMENTS}

This work is supported by the Department of Energy, Office of Basic Energy Sciences, Division of Materials Sciences and Engineering, under Contract No. DE-AC0276SF00515, and by the Swedish National Research Council. This research was partly carried out at the Stanford Synchrotron Radiation Lightsource, a National User Facility operated by Stanford University on behalf of the U.S. Department of Energy, Office of Basic Energy Sciences.

${ }^{1}$ M. A. Henderson, Surf. Sci. Rep. 46, 1 (2002).

${ }^{2}$ T. Schiros, K. J. Andersson, L. G. M. Pettersson, A. Nilsson, and H. Ogasawara, J. Electron Spectrosc. Relat. Phenom. 177, 85-98 (2010).

${ }^{3}$ A. Michaelides, V. A. Ranea, P. L. de Andres, and D. A. King, Phys. Rev. Lett. 90, 216102 (2003).

${ }^{4}$ A. Hodgson and S. Haq, Surf. Sci. Rep. 64, 381 (2009).

${ }^{5}$ J. Cerdá et al., Phys. Rev. Lett. 93, 116101 (2004).

${ }^{6}$ T. Mitsui, M. K. Rose, E. Fomin, D. F. Ogletree, and M. Salmerón, Science 297, 1850-1852 (2002).

${ }^{7}$ C. Nöbl, C. Benndorf, and T. E. Madey, Surf. Sci. 157, 29-42 (1985).

${ }^{8}$ B. W. Callen, K. Griffiths, and P. R. Norton, Phys. Rev. Lett. 66, 1634-1637 (1991).

${ }^{9}$ A. Michaelides and K. Morgenstern, Nature Mater. 6, 597 (2007).

${ }^{10}$ K. Morgenstern and J. Nieminen, Phys. Rev. Lett. 88, 066102 (2002).

${ }^{11}$ D. Nordlund et al., Phys. Rev. B 80, 233404 (2009).

${ }^{12}$ T. Yamada, S. Tamamori, H. Okuyama, and T. Aruga, Phys. Rev. Lett. 96, 036105 (2006).

${ }^{13}$ T. Kumagai, H. Okuyama, S. Hatta, T. Aruga, and I. Hamada, J. Chem. Phys. 134, 024703 (2011).

${ }^{14}$ J. Carrasco et al., Nature Mater. 8, 427-431 (2009).

${ }^{15}$ K. Andersson et al., Surf. Sci. Lett. 585, L183-L189 (2005).

${ }^{16}$ J. Ren and S. Meng, J. Am. Chem. Soc. 128, 9282 (2006).

${ }^{17}$ N. A. Koryabkina, A. A. Phatak, W. F. Ruettinger, R. J. Farrauto, and F. H. Ribeiro, J. Catal. 217, 233-239 (2004).

${ }^{18}$ A. Ya. Rozovskii, Kinet. Catal. 44, 360 (2003).

${ }^{19}$ D. S. Newsome, Catal. Rev.-Sci. Eng. 21, 275-318 (1980).

${ }^{20}$ H. Gränicher, Z. Kristallogr. 110, 432-471 (1958).

${ }^{21}$ M. Forster, R. Raval, A. Hodgson, J. Carrasco, and A. Michaelides, Phys. Rev. Lett. 106, 046103 (2011).

${ }^{22}$ J. Stöhr, NEXAFS Spectroscopy (Springer-Verlag, 1992).

${ }^{23}$ T. Schiros et al., J. Chem. Phys. 132, 094701 (2010).

${ }^{24}$ StoBe-deMon, version 5.3, deMon Software, Stockholm-Berlin, 2005.

${ }^{25}$ L. Triguero, L. G. M. Pettersson, and H. Ågren, Phys. Rev. B 58, 8097 (1998).

${ }^{26}$ M. Leetmaa, M. P. Ljungberg, A. P. Lyubartsev, A. Nilsson, and L. G. M. Pettersson, J. Electron Spectrosc. Relat. Phenom. 177, 135-157 (2010).

${ }^{27}$ T. Schiros et al., Chem. Phys. Lett. 429, 415 (2006).

${ }^{28}$ C. Kolczewski et al., J. Chem. Phys. 115, 6426-6437 (2001).

${ }^{29}$ D. Nordlund et al., Chem. Phys. Lett. 395, 161-165 (2004).

${ }^{30}$ M. Nakamura and M. Ito, Chem. Phys. Lett. 404, 346-350 (2005).

${ }^{31}$ S. Haq, C. Clay, G. R. Darling, G. Zimnitas, and A. Hodgson, Phys. Rev. B 73, 115414 (2006).

${ }^{32}$ D. Nordlund et al., Phys. Rev. Lett. 99, 217406 (2007).

${ }^{33}$ M. Cavalleri, H. Ogasawara, L. G. M. Pettersson, and A. Nilsson, Chem. Phys. Lett. 364, 363 (2002).

${ }^{34}$ S. Myneni et al., J. Phys.: Condens. Matter 14, L213-L219 (2002).

${ }^{35}$ P. Wernet et al., Science 304, 995-999 (2004).

${ }^{36}$ A. Nilsson et al., J. Electron Spectrosc. Relat. Phenom. 177, 99-129 (2010).

${ }^{37}$ H. Ogasawara et al., Phys. Rev. Lett. 89, 276102 (2002).

${ }^{38}$ See supplementary material at http://dx.doi.org/10.1063/1.4809680 for Cartesian coordinates for the structure models used in Figure 3.

${ }^{39}$ R. Smoluchowski, Phys. Rev. 60, 661 (1941).

${ }^{40}$ K. Gustafsson, G. S. Karlberg, and S. Andersson, J. Chem. Phys. 127, 194708 (2007).

${ }^{41}$ K. Svensson and S. Andersson, Phys. Rev. Lett. 78, 2016 (1997). 
${ }^{42}$ K. Gustafsson and S. Andersson, J. Chem. Phys. 120, 7750 (2004).

${ }^{43}$ K. Gustafsson and S. Andersson, J. Chem. Phys. 125, 044717 (2006).

${ }^{44}$ P. Hollins, K. J. Davies, and J. Pritchard, Surf. Sci. 138, 75-83 (1984).

${ }^{45}$ T. Schiros et al., J. Phys. Chem. C 111, 15003-15012 (2007).

${ }^{46}$ M. Tatarkhanov et al., J. Chem. Phys. 129, 154109 (2008).

${ }^{47}$ T. Pylkkänen et al., J. Phys. Chem. B 114, 3804 (2010).

${ }^{48}$ D. Prendergast, and G. Galli, Phys. Rev. Lett. 96, 215502 (2006).

${ }^{49}$ M. Iannuzzi, J. Chem. Phys. 128, 204506 (2008).

${ }^{50}$ L. Z. Kong, X. F. Wu, and R. Car, Phys. Rev. B 86, 134203 (2012).

${ }^{51}$ W. Chen, X. Wu, and R. Car, Phys. Rev. Lett. 105, 017802 (2010).

${ }^{52}$ C. D. Cappa, J. D. Smith, B. M. Messer, R. C. Cohen, and R. J. Saykally, J. Phys. Chem. A 111, 4776-4785 (2007).

${ }^{53}$ J. Stöhr, F. Sette, and A. L. Johnson, Phys. Rev. Lett. 53, 1684 (1984).
${ }^{54}$ M. Odelius, M. Cavalleri, A. Nilsson, and L. G. M. Pettersson, Phys. Rev. B 73, 024205 (2006).

${ }^{55}$ K. S. Kim et al., Phys. Rev. Lett. 76, 956 (1996).

${ }^{56}$ L. Kevan, Acc. Chem. Res. 14, 138 (1981).

${ }^{57}$ M. Leetmaa et al., J. Chem. Phys. 125, 244510 (2006).

${ }^{58}$ A. K. Soper, J. Phys.: Condens. Matter 17, S3273-S3282 (2005).

${ }^{59}$ X.-Z. Li, M. I. J. Probert, A. Alavi, and A. Michaelides, Phys. Rev. Lett. 104, 066102 (2010).

${ }^{60}$ X.-Z. Li, B. Walker, and A. Michaelides, Proc. Natl. Acad. Sci. U.S.A. 108, 6369-6373 (2011).

${ }^{61}$ T. Yamada, S. Tamamori, H. Okuyama, and T. Aruga, J. Surf. Sci. Soc. Jpn. 27, 455-460 (2006).

${ }^{62}$ J. H. Hunt, P. Guyot-Sionnest, and Y. R. Shen, Chem. Phys. Lett. 133, 189192 (1987). 